\title{
Real time motorcycle image detections on field programmable gate array
}

\begin{abstract}
Development of real time motorcycle image detections on field programmable architecture is presented in this paper. This paper describes a process to generate a bounding box and analyzes the system performance in tracking the image and hardware utilize. This paper also discussed on image processing algorithm used and embedded techniques involved. The performance evaluation results also indicated and shows that development design meet the requirement.
\end{abstract}

Keyword: Bounding box; Critical condition; Driving assistance system; FPGA; Motorcycle 